\title{
Nonadditivity of critical Casimir forces
}

\author{
Agnese Callegari \\ Soft Matter Lab, Department of Physics, Bilkent University, Ankara 06800, Turkey \\ callegari@fen.bilkent.edu.tr \\ Sathyanarayana Paladugu, Yazgan Tuna, Lukas Barth \\ Soft Matter Lab, Department of Physics, Bilkent University, Ankara 06800, Turkey \\ Siegfried Dietrich \\ Max-Planck-Institut für Intelligente Systeme, Heisenbergstr. 3, D-70569 Stuttgart, Germany \\ Institut für Theoretische Physik, Universität Stuttgart, Pfaffenwaldring 57, D-70569 Stuttgart, Germany \\ Andrea Gambassi \\ SISSA - International School for Advanced Studies and INFN, via Bonomea 265, 34136 Trieste, Italy \\ Giovanni Volpe \\ Soft Matter Lab, Department of Physics, Bilkent University, Ankara 06800, Turkey \\ Göteborgs Universitet, Institutionen för Fysik, 41296 Göteborg, Sweden
}

\begin{abstract}
We provide the first experimental evidence of nonadditivity for critical Casimir forces: the force that two colloidal particles exert together on a third one differs from the sum of the forces they exert separately.

OCIS codes: (350.4855) Optical tweezers or optical manipulation; (350.0350) Other areas of optics
\end{abstract}

In soft condensed matter physics, effective interactions often emerge due to the spatial confinement of fluctuating fields. For instance, microscopic particles dissolved in a binary liquid mixture are subject to critical Casimir forces whenever their surfaces confine the thermal fluctuations of the order parameter of the solvent close to its critical demixing point. These forces are theoretically predicted to be nonadditive on the scale set by the bulk correlation length of the fluctuations. Here, we provide direct experimental evidence of this fact by reporting the measurement of the associated many-body effects. We consider three colloidal particles in optical traps[1] and observe that the critical Casimir force exerted on one of them by the other two differs from the sum of the forces they exert separately (Fig. 1). This three-body effect depends sensitively on the distance from the critical point and on the preference of the colloid surfaces for one of the components of the mixture[2].
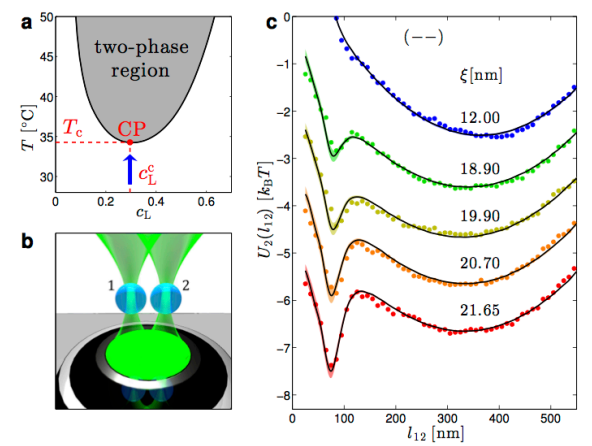

d

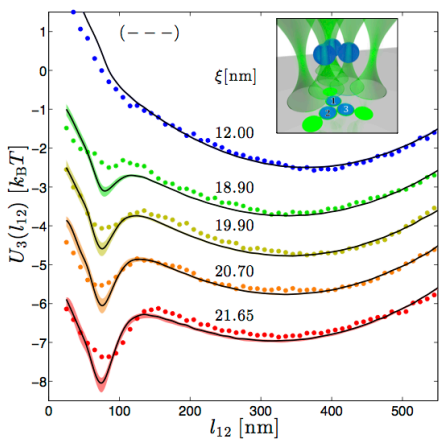

Figure 1: (a) phase-diagram of the mixture water-lutidine; (b) pictorial representation for the trapping; (c) two-body potentials as a function of the temperature; (d) two-body potentials in presence of a third near particle as a function of the temperature: experimental (dot) and simulations (line). From [2]

[1] P. H. Jones, O. M. Maragò \& G. Volpe. Optical Tweezers: Principles and Applications. Cambridge University Press (2015). ISBN:9781107051164

[2] S. Paladugu, A. Callegari, Y. Tuna, L. Barth, S. Dietrich, A. Gambassi, and G. Volpe, "Nonadditivity of critical Casimir forces", Nat. Comm. 7, 11403 (2016). 